\title{
NONLINEAR ERGODIC THEOREMS FOR A SEMITOPOLOGICAL SEMIGROUP OF NON-LIPSCHITZIAN MAPPINGS WITHOUT CONVEXITY
}

\author{
G. LI AND J. K. KIM \\ Received 2 February 1999
}

Let $G$ be a semitopological semigroup, $C$ a nonempty subset of a real Hilbert space $H$, and $\mathfrak{s}=\left\{T_{t}: t \in G\right\}$ a representation of $G$ as asymptotically nonexpansive type mappings of $C$ into itself. Let $L(x)=\left\{z \in H: \inf _{s \in G} \sup _{t \in G}\left\|T_{t s} x-z\right\|=\inf _{t \in G}\left\|T_{t} x-z\right\|\right\}$ for each $x \in C$ and $L(\Im)=\bigcap_{x \in C} L(x)$. In this paper, we prove that $\bigcap_{s \in G} \overline{\operatorname{conv}}\left\{T_{t s} x\right.$ : $t \in G\} \bigcap L(\Im)$ is nonempty for each $x \in C$ if and only if there exists a unique nonexpansive retraction $P$ of $C$ into $L(\Im)$ such that $P T_{s}=P$ for all $s \in G$ and $P(x) \in \overline{\operatorname{conv}}\left\{T_{s} x: s \in G\right\}$ for every $x \in C$. Moreover, we prove the ergodic convergence theorem for a semitopological semigroup of non-Lipschitzian mappings without convexity.

\section{Introduction and preliminaries}

Let $H$ be a Hilbert space with norm $\|\cdot\|$ and inner product $(\cdot, \cdot)$. Let $G$ be a semitopological semigroup, that is, a semigroup with a Hausdorff topology such that for each $s \in G$ the mappings $s \mapsto s \cdot t$ and $s \mapsto t \cdot s$ of $G$ into itself are continuous. Let $C$ be a nonempty subset of $H$ and let $\mathfrak{s}=\left\{T_{t}: t \in G\right\}$ be a semigroup on $C$, that is, $T_{s t}(x)=T_{s} T_{t}(x)$ for all $s, t \in G$ and $x \in C$. Recall that a semigroup $\Im$ is said to be

(a) nonexpansive if $\left\|T_{t} x-T_{t} y\right\| \leq\|x-y\|$ for $x, y \in C$ and $t \in G$.

(b) asymptotically nonexpansive [6] if there exists a function $k: G \mapsto[0, \infty)$ with $\inf _{s \in G} \sup _{t \in G} k_{t s} \leq 1$ such that $\left\|T_{t} x-T_{t} y\right\| \leq k_{t}\|x-y\|$ for $x, y \in C$ and $t \in G$.

(c) of asymptotically nonexpansive type [6] if for each $x$ in $C$, there is a function $r(\cdot, x): G \mapsto[0, \infty)$ with $\inf _{s \in G} \sup _{t \in G} r(t s, x)=0$ such that $\left\|T_{t} x-T_{t} y\right\| \leq \| x-$ $y \|+r(t, x)$ for all $y \in C$ and $t \in G$.

It is easily seen that $(\mathrm{a}) \Rightarrow(\mathrm{b}) \Rightarrow(\mathrm{c})$ and that both the inclusions are proper (cf. [6, page 112]).

Baillon [1] proved the first nonlinear mean ergodic theorem for nonexpansive mappings in a Hilbert space: let $C$ be a nonempty closed convex subset of a Hilbert space $H$ and $T$ a nonexpansive mapping of $C$ into itself. If the set $F(T)$ of fixed points of $T$

Copyright $\odot 1999$ Hindawi Publishing Corporation

Abstract and Applied Analysis 4:1 (1999) 49-59

1991 Mathematics Subject Classification: 47H09, 47H10, 47H20

URL: http://aaa.hindawi.com/volume-4/S1085337599000056.html 
is nonempty, then for each $x \in C$, the Cesáro means

$$
S_{n}(x)=\frac{1}{n} \sum_{k=0}^{n-1} T^{k} x
$$

converge weakly as $n \rightarrow \infty$ to a point of $F(T)$. In this case, putting $y=P x$ for each $x \in C, P$ is a nonexpansive retraction of $C$ onto $F(T)$ such that $P T=T P=P$ and $P x \in \overline{\operatorname{conv}}\left\{T^{n} x: n=0,1,2, \ldots\right\}$ for each $x \in C$, where $\overline{\operatorname{conv}} A$ is the closure of the convex hull of $A$. The analogous results are given for nonexpansive semigroups on $C$ by Baillon [2] and Breźis-Browder [3]. In [10], Mizoguchi-Takahashi proved a nonlinear ergodic retraction theorem for Lipschitzian semigroups by using the notion of submean. Recently, Li and Ma $[8,9]$ proved the nonlinear ergodic retraction theorems for nonLipschitzian semigroups in a Banach space without using the notion of submean. Also, in 1992, Takahashi [13] proved the ergodic theorem for nonexpansive semigroups on condition that $\bigcap_{s \in G} \overline{\operatorname{conv}}\left\{T_{s t} x: t \in G\right\} \subset C$ for some $x \in C$.

In this paper, without using the concept of submean, we prove nonlinear ergodic theorem for semitopological semigroup of non-Lipschitzian mappings without convexity in a Hilbert space. We first prove that if $C$ is a nonempty subset of a Hilbert space $H, G$ a semitopological semigroup, and $\mathfrak{s}=\left\{T_{t}: t \in G\right\}$ a representation of $G$ as asymptotically nonexpansive type mappings of $C$ into itself, then $\bigcap_{s \in G} \overline{\operatorname{conv}}\left\{T_{t s} x\right.$ : $t \in G\} \bigcap L(\Im)$ is nonempty for each $x \in C$ if and only if there exists a unique nonexpansive retraction $P$ of $C$ into $L(\Im)$ such that $P T_{s}=P$ for all $s \in G$ and $P x$ is in the closed convex hull of $\left\{T_{s} x: s \in G\right\}$, where $L(x)=\left\{z: \inf _{s \in G} \sup _{t \in G} \| T_{t s} x-\right.$ $\left.z\left\|=\inf _{t \in G}\right\| T_{t} x-z \|\right\}$ and $L(\mathfrak{I})=\bigcap_{x \in C} L(x)$. By using this result, we also prove the ergodic convergence theorem for semitopological semigroup of non-Lipschitzian mapping without convexity. Our results are generalizations and improvements of the previously known results of Brézis-Browder [3], Hirano-Takahashi [4], MizoguchiTakahashi [10], Takahashi-Zhang [14], and Takahashi [11, 12, 13] in many directions. Further, it is safe to say that in the results $[1,2,3,4,5,7,10,11,12,13,14]$, many key conditions are not necessary.

\section{Ergodic convergence theorems}

Throughout this paper, we assume that $C$ is a nonempty subset of a real Hilbert space $H, G$ a semitopological semigroup, and $\mathfrak{s}=\left\{T_{t}: t \in G\right\}$ an asymptotically nonexpansive type semigroup on $C$. For each $x \in C$, define $L(x)$ and $L(\Im)$ by

$$
L(x)=\left\{z: \inf _{s \in G} \sup _{t \in G}\left\|T_{t s} x-z\right\|=\inf _{t \in G}\left\|T_{t} x-z\right\|\right\}, \quad L(\Im)=\bigcap_{x \in C} L(x),
$$

respectively. We denote $F(\Im)$ by the set $\left\{x \in C: T_{s}(x)=x\right.$ for all $\left.s \in G\right\}$ of common fixed point of $\mathfrak{s}$. We begin with the following lemma.

Lemma 2.1. Let $C$ be a nonempty subset of a Hilbert space $H$ and $\mathfrak{s}=\left\{T_{t}: t \in G\right\}$ an asymptotically nonexpansive type semigroup on $C$. Then $F(\Im) \subset L(\Im)$. 
Proof. Let $x \in C$ and $f \in F(\mathfrak{I})$. Since $\mathfrak{s}$ is asymptotically nonexpansive type, for an arbitrary $\varepsilon>0$, there exists $s_{0} \in G$ such that for all $t \in G$

$$
r\left(t s_{0}, f\right)<\varepsilon
$$

Hence, for each $a \in G$,

$$
\begin{aligned}
\inf _{s \in G} \sup _{t \in G}\left\|T_{t s} x-f\right\| & \leq \sup _{t \in G}\left\|T_{t s_{0} a} x-f\right\| \leq \sup _{t \in G}\left(\left\|T_{a} x-f\right\|+r\left(t s_{0}, f\right)\right) \\
& \leq\left\|T_{a} x-f\right\|+\varepsilon .
\end{aligned}
$$

Since $\varepsilon>0$ is arbitrary, we have $\inf _{s \in G} \sup _{t \in G}\left\|T_{t s} x-f\right\| \leq \inf _{t \in G}\left\|T_{t} x-f\right\|$. Therefore, $f \in L(x)$. This completes the proof.

Remark 2.2. It is not easy to prove that $F(\mathfrak{I})$ is nonempty when $C$ is not a convex subset. However, we can show that $L(\mathfrak{I})$ is nonempty under some conditions and it is important for the ergodic convergence theorem.

The following proposition plays a crucial role in the proof of our main theorems in this paper.

Proposition 2.3. Let $G$ be a semitopological semigroup, $C$ a nonempty subset of a Hilbert space $H$, and $\Im=\left\{T_{t}: t \in G\right\}$ an asymptotically nonexpansive type semigroup on $C$. Then, for every $x \in C$, the set

$$
\bigcap_{s \in G} \overline{\operatorname{conv}}\left\{T_{t s} x: t \in G\right\} \bigcap L(x),
$$

consists of at most one point.

Proof. Let $u, v \in \bigcap_{s \in G} \overline{\operatorname{conv}}\left\{T_{t s} x: t \in G\right\} \bigcap L(x)$, without loss of generality, we assume that

$$
\inf _{t \in G}\left\|T_{t} x-u\right\|^{2} \leq \inf _{t \in G}\left\|T_{t} x-v\right\|^{2} .
$$

Now, for each $t, s \in G$, since

$$
\|u-v\|^{2}+2\left(T_{t s} x-u, u-v\right)=\left\|T_{t s} x-v\right\|^{2}-\left\|T_{t s} x-u\right\|^{2},
$$

we have

$$
\begin{aligned}
\|u-v\|^{2}+2 \inf _{t \in G}\left(T_{t s} x-u, u-v\right) & \geq \inf _{t \in G}\left\|T_{t s} x-v\right\|^{2}-\sup _{t \in G}\left\|T_{t s} x-u\right\|^{2} \\
& \geq \inf _{t \in G}\left\|T_{t} x-v\right\|^{2}-\sup _{t \in G}\left\|T_{t s} x-u\right\|^{2} .
\end{aligned}
$$

From $u \in L(x)$, we have

$$
\begin{aligned}
\|u-v\|^{2}+2 \sup _{s \in G} \inf _{t \in G}\left(T_{t s} x-u, u-v\right) & \geq \inf _{t \in G}\left\|T_{t} x-v\right\|^{2}-\inf _{s \in G} \sup _{t \in G}\left\|T_{t s} x-u\right\|^{2} \\
& =\inf _{t \in G}\left\|T_{t} x-v\right\|^{2}-\inf _{t \in G}\left\|T_{t} x-u\right\|^{2} \geq 0 .
\end{aligned}
$$


Therefore, for $\varepsilon>0$ there is an $s_{1} \in G$ such that

$$
\|u-v\|^{2}+2\left(T_{t s_{1}} x-u, u-v\right)>-\varepsilon \quad \forall t \in G .
$$

From $v \in \overline{\operatorname{conv}}\left\{T_{t s_{1}} x: t \in G\right\}$, we have

$$
\|u-v\|^{2}+2(v-u, u-v) \geq-\varepsilon .
$$

This inequality implies that $\|u-v\|^{2} \leq \varepsilon$. Since $\varepsilon>0$ is arbitrary, we have $u=v$. This completes the proof.

Remark 2.4. In the Takahashi-Zhang's result [14], it is assumed that $C$ is a closed convex subset, $G$ a reversible semigroup, and $\Im$ an asymptotically nonexpansive semigroup. Proposition 2.3 shows those key conditions are not necessary.

Let $m(G)$ be the Banach space of all bounded real-valued functions on a semitopological semigroup $G$ with the supremum norm and let $X$ be a subspace of $m(G)$ containing constants. Then, an element $\mu$ of $X^{*}$ (the dual space of $X$ ) is called a mean on $X$ if $\|\mu\|=\mu(1)=1$. Let $\mu$ be a mean on $X$ and $f \in X$. Then, according to time and circumstances, we use $\mu_{t}(f(t))$ instead of $\mu(f)$. For each $s \in G$ and $f \in m(G)$, we define elements $l_{s} f$ and $r_{s} f$ in $m(G)$ given by $\left(l_{s} f\right)(t)=f(s t)$ and $\left(r_{s} f\right)(t)=f(t s)$ for all $t \in G$, respectively.

Throughout the rest of this section, let $X$ be a subspace of $m(G)$ containing constants invariant under $l_{s}$ and $r_{s}$ for each $s \in G$. Furthermore, suppose that for each $x \in C$ and $y \in H$, a function $f(t)=\left\|T_{t} x-y\right\|^{2}$ is in $X$. For $\mu \in X^{*}$, we define the value $\mu_{t}\left(T_{t} x, y\right)$ of $\mu$ at this function. By Riesz theorem, there exists a unique element $\Im_{\mu} x$ in $X$ such that

$$
\mu_{t}\left(T_{t} x, y\right)=\left(\Im_{\mu} x, y\right) \quad \forall y \in H .
$$

Lemma 2.5. Suppose that $X$ has an invariant mean $\mu$. Then we have

$$
\bigcap_{s \in G} \overline{\operatorname{conv}}\left\{T_{t s} x: t \in G\right\} \bigcap L(x)=\left\{\Im_{\mu} x\right\} \quad \text { for every } x \in C .
$$

Further, if $T_{t}$ is continuous for each $t \in G$ and $\bigcap_{s \in G} \overline{\operatorname{conv}}\left\{T_{s t} x: t \in G\right\} \subset C$ for some $x \in C$, then $\mathfrak{\Im}_{\mu} x \in F(\mathfrak{\Im})$.

Proof. Since $\mu$ is an invariant mean, it is easy to show that $\Im_{\mu} x \in \bigcap_{s \in G} \overline{\operatorname{conv}}\left\{T_{t s} x\right.$ : $t \in G\}$ for each $x \in C$. By Proposition 2.3, it is enough to prove that $\mathfrak{\Im}_{\mu} x \in L(x)$ for each $x \in C$. To this end, let $\varepsilon>0$, since $\mathfrak{s}$ is an asymptotically nonexpansive type semigroup, for each $t \in G$ there is an $h_{t} \in G$ such that for each $h \in G$,

$$
r\left(h h_{t}, T_{t} x\right)<\varepsilon .
$$

Put $M=\sup _{t, s \in G}\left\|T_{t} x-T_{s} x\right\|$, then we have

$$
\begin{aligned}
\left\|T_{h h_{t} t} x-\Im_{\mu} x\right\|^{2}-\left\|T_{t} x-\Im_{\mu} x\right\|^{2} & =\mu_{s}\left(\left\|T_{h h_{t} t} x-T_{s} x\right\|^{2}-\left\|T_{t} x-T_{s} x\right\|^{2}\right) \\
& =\mu_{s}\left(\left\|T_{h h_{t} t} x-T_{h h_{t} s} x\right\|^{2}-\left\|T_{t} x-T_{s} x\right\|^{2}\right) \\
& \leq 2 M \varepsilon \text { for each } h \in G .
\end{aligned}
$$


Hence, we have

$$
\inf _{s \in G} \sup _{h \in G}\left\|T_{h s} x-\Im_{\mu} x\right\|^{2} \leq\left\|T_{t} x-\Im_{\mu} x\right\|^{2}+2 M \varepsilon \quad \forall t \in G
$$

Since $\varepsilon>0$ is arbitrary, we have $\mathfrak{\Im}_{\mu} x \in L(x)$. Finally, suppose that $\bigcap_{s \in G} \overline{\operatorname{conv}}\left\{T_{s t} x\right.$ : $t \in G\} \subset C$ and each $T_{t}$ is continuous from $C$ into itself. Then, we can easily prove that $\mathfrak{\Im}_{\mu} x \in \bigcap_{s \in G} \overline{\operatorname{conv}}\left\{T_{s t} x: t \in G\right\}$ and hence we have $\mathfrak{\Im}_{\mu} x \in C$. For each $h \in G$ and $\varepsilon \in(0,1)$, there exists $0<\delta<\varepsilon$ such that $\left\|T_{h} y-T_{h} \mathfrak{\Im}_{\mu} x\right\|<\varepsilon$ whenever $y \in C$ and $\left\|y-\mathfrak{\Im}_{\mu} x\right\| \leq \delta$. Since $\mathfrak{s}$ is an asymptotically nonexpansive type semigroup, there is $s_{0} \in G$ such that

$$
r\left(t s_{0}, \Im_{\mu} x\right)<\frac{1}{2\left(M_{1}+1\right)} \delta^{2} \quad \forall t \in G,
$$

where $M_{1}=\sup _{t \in G}\left\|T_{t} x-\mathfrak{\Im}_{\mu} x\right\|$. Then for each $t, s \in G$, we have

$$
\begin{aligned}
& \left\|T_{s s_{0}} \mathfrak{\Im}_{\mu} x-\mathfrak{\Im}_{\mu} x\right\|^{2}+2\left(T_{t} x-\Im_{\mu} x, \mathfrak{\Im}_{\mu} x-T_{s s_{0}} \mathfrak{\Im}_{\mu} x\right) \\
& \quad=\left\|T_{t} x-T_{s s_{0}} \mathfrak{\Im}_{\mu} x\right\|^{2}-\left\|T_{t} x-\mathfrak{\Im}_{\mu} x\right\|^{2} \\
& \quad=\left\|T_{s s_{0} t} x-T_{s s_{0}} \mathfrak{\Im}_{\mu} x\right\|^{2}-\left\|T_{t} x-\Im_{\mu} x\right\|^{2}-\left\|T_{s s_{0} t} x-T_{s s_{0}} \Im_{\mu} x\right\|^{2}+\left\|T_{t} x-T_{s s_{0}} \mathfrak{\Im}_{\mu} x\right\|^{2} \\
& \quad \leq \delta^{2}-\left\|T_{s s_{0} t} x-T_{s s_{0}} \mathfrak{\Im}_{\mu} x\right\|^{2}+\left\|T_{t} x-T_{s s_{0}} \mathfrak{s}_{\mu} x\right\|^{2} .
\end{aligned}
$$

It follows that

$$
\left\|T_{s s_{0}} \mathfrak{\Im}_{\mu} x-\mathfrak{\Im}_{\mu} x\right\| \leq \delta \quad \forall s \in G
$$

This implies that

$$
\left\|T_{h} \mathfrak{\Im}_{\mu} x-\mathfrak{\Im}_{\mu} x\right\| \leq\left\|T_{h} \mathfrak{\Im}_{\mu} x-T_{h} T_{s s_{0}} \mathfrak{\Im}_{\mu} x\right\|+\left\|T_{h s_{0}} \mathfrak{\Im}_{\mu} x-\mathfrak{\Im}_{\mu} x\right\|<2 \varepsilon .
$$

Since $\varepsilon>0$ is arbitrary, we have $T_{h} \mathfrak{s}_{\mu} x=\mathfrak{s}_{\mu} x$. This completes the proof.

Now, we prove a nonlinear ergodic theorem for asymptotically nonexpansive type semigroups without convexity. Before doing this, we give a definition concerning means. Let $\left\{\mu_{\alpha}: \alpha \in A\right\}$ be a net of means on $X$, where $A$ is a directed set. Then $\left\{\mu_{\alpha}: \alpha \in A\right\}$ is said to be asymptotically invariant if for each $f \in X$ and $s \in G$,

$$
\mu_{\alpha}(f)-\mu_{\alpha}\left(l_{s} f\right) \longrightarrow 0, \quad \mu_{\alpha}(f)-\mu_{\alpha}\left(r_{s} f\right) \longrightarrow 0
$$

TheORem 2.6. Let $C$ be a nonempty subset of a Hilbert space $H, X$ an invariant subspace of $m(G)$ containing constants, and $\mathfrak{s}=\left\{T_{t}: t \in G\right\}$ an asymptotically nonexpansive type semigroup on $C$. If for each $x \in C$ and $y \in H$, the function $f$ on $G$ defined by $f(t)=\left\|T_{t} x-y\right\|^{2}$ belong to $X$, then for an asymptotically invariant net $\left\{\mu_{\alpha}: \alpha \in A\right\}$ on $X$, the net $\left\{\Im_{\mu_{\alpha}} x\right\}_{\alpha \in A}$ converges weakly to an element $x_{0} \in L(x)$. 
Further, if $T_{t}$ is continuous for each $t \in G$ and $\bigcap_{s \in G} \overline{\operatorname{conv}}\left\{T_{s t} x: t \in G\right\} \subset C$, then $x_{0} \in F(\Im)$.

Proof. Let $W$ be the set of all weak limit points of subnet of the net $\left\{\Im_{\mu_{\alpha}} x: \alpha \in A\right\}$. By Proposition 2.3, it is enough to prove that

$$
W \subset \bigcap_{s \in G} \overline{\operatorname{conv}}\left\{T_{t s} x: t \in G\right\} \bigcap L(x) .
$$

To show this, let $z \in W$ and let $\left\{\Im_{u_{\alpha_{\beta}}} x\right\}$ be a subnet of $\left\{\Im_{\mu_{\alpha}} x\right\}$ such that $\left\{\Im_{\mu_{\alpha_{\beta}}} x\right\}$ converges weakly to $z$. Now, without loss of generality, we can suppose that $\left\{\Im_{\mu_{\alpha_{\beta}}} x\right\}$ converges weakly* to $\mu \in X^{*}$. It is easily seen that $\mu$ is an invariant mean on $X$ and then Lemma 2.5 implies that $z=\mathfrak{s}_{\mu} x \in \bigcap_{s \in G} \overline{\operatorname{conv}}\left\{T_{t s} x: t \in G\right\} \bigcap L(x)$. This completes the proof.

Let $C(G)$ be the Banach space of all bounded continuous real-valued functions on $G$ and let $R U C(G)$ be the space of all bounded right uniformly continuous functions on $G$, that is, all $f \in C(G)$ such that the mapping $s \mapsto r_{s} f$ is continuous. Then $\operatorname{RUC}(G)$ is a closed subalgebra of $C(G)$ containing constants and invariant under $l_{s}$ and $r_{s}$.

As a direct consequence of Theorem 2.6, we obtain the following corollary.

Corollafry 2.7 (see [13]). Let $C$ be a nonempty subset of a Hilbert space $H$ and let $G$ be a semitopological semigroup such that $R U C(G)$ has an invariant mean. Let $\mathfrak{s}=\left\{T_{t}: t \in G\right\}$ be a nonexpansive semigroup on $C$ such that $\left\{T_{t} x: t \in G\right\}$ is bounded and $\bigcap_{s \in G} \overline{\operatorname{conv}}\left\{T_{s t} x: t \in G\right\} \subset C$ for some $x \in C$. Then, $F(\Im) \neq \emptyset$. Further, for an asymptotically invariant net $\left\{\mu_{\alpha}\right\}_{\alpha \in A}$ of means on $\operatorname{RUC}(G)$, the net $\left\{\mathfrak{\Im}_{\mu_{\alpha}}\right\}_{\alpha \in A}$, converges weakly to an element $x_{0} \in F(\mathfrak{I})$.

Remark 2.8. For the proof of Corollary 2.7, Takahashi [13] used the condition $\bigcap_{s \in G}$ $\overline{\operatorname{conv}}\left\{T_{s t} x: t \in G\right\} \subset C$. But, from Theorem 2.6, we can prove the result without this condition except proving the fact that the weak limit of $\left\{\mathfrak{\Im}_{\mu_{\alpha}} x\right\}$ is in $F(\mathfrak{s})$.

\section{Nonexpansive retractions}

In this section, we prove an ergodic retraction theorem for a semitopological semigroup of asymptotically nonexpansive type mappings without convexity.

THEOREM 3.1. Let $C$ be a nonempty subset of a Hilbert space $H$ and let $\mathfrak{s}=\left\{T_{t}: t \in G\right\}$ be a semitopological semigroup of asymptotically nonexpansive type mappings on $C$ such that $L(\mathfrak{I}) \neq \emptyset$. Then the following statements are equivalent:

(a) $\bigcap_{s \in G} \overline{\operatorname{conv}}\left\{T_{t s} x: t \in G\right\} \cap L(\Im) \neq \varnothing$ for each $x \in C$.

(b) There is a unique nonexpansive retraction $P$ of $C$ into $L(\Im)$ such that $P T_{t}=P$ for every $t \in G$ and $P x \in \overline{\operatorname{conv}}\left\{T_{t} x: t \in G\right\}$ for every $x \in C$.

Proof. (b) $\Rightarrow$ (a). Let $x \in C$, then $P x \in L(\Im)$. Also $P x \in \bigcap_{s \in G} \overline{\operatorname{conv}}\left\{T_{t s} x: t \in G\right\}$. In fact, for each $s \in G, P x=P T_{s} x \in \overline{\operatorname{conv}}\left\{T_{t} T_{s} x: t \in G\right\}=\overline{\operatorname{conv}}\left\{T_{t s} x: t \in G\right\}$. 
(a) $\Rightarrow$ (b). Let $x \in C$. Then by Proposition 2.3, $\bigcap_{s \in G} \overline{\operatorname{conv}}\left\{T_{t s} x: t \in G\right\} \bigcap L(\mathfrak{I})$ contains exactly one point $P x$. For each $a \in G$, we have

$$
\begin{aligned}
\left\{P T_{a} x\right\} & =\bigcap_{s \in G} \overline{\operatorname{conv}}\left\{T_{t s a} x: t \in G\right\} \bigcap L(\Im) \\
& \supseteq \bigcap_{s \in G} \overline{\operatorname{conv}}\left\{T_{t s} x: t \in G\right\} \bigcap L(\Im)=\{P x\}
\end{aligned}
$$

and hence we have $P T_{a}=P$ for every $a \in G$.

Finally, we have to show that $P$ is nonexpansive. Let $x, y \in C$ and $0<\lambda<1$. Then for any $\varepsilon>0$, there exists $s_{1} \in G$ such that

$$
\sup _{t \in G}\left\|T_{t s_{1}} x-P y\right\| \leq \inf _{t \in G}\left\|T_{t} x-P y\right\|+\varepsilon,
$$

from $P y \in L(\Im)$. Hence, we have

$$
\begin{aligned}
& \left\|\lambda T_{t s s_{1}} x+(1-\lambda) P x-P y\right\|^{2} \\
& \quad=\left\|\lambda\left(T_{t s s_{1}} x-P y\right)+(1-\lambda)(P x-P y)\right\|^{2} \\
& \quad=\lambda\left\|T_{t s s_{1}} x-P y\right\|^{2}+(1-\lambda)\|P x-P y\|^{2}-\lambda(1-\lambda)\left\|T_{t s s_{1}} x-P x\right\|^{2} \\
& \quad \leq \lambda\left(\left\|T_{a b} x-P y\right\|+\varepsilon\right)^{2}+(1-\lambda)\|P x-P y\|^{2}-\lambda(1-\lambda) \inf _{t \in G}\left\|T_{t} x-P x\right\|^{2},
\end{aligned}
$$

for each $t, s, a, b \in G$. Since $\varepsilon>0$ is arbitrary, this implies

$$
\begin{aligned}
& \inf _{s \in G} \sup _{t \in G}\left\|\lambda T_{t s} x+(1-\lambda) P x-P y\right\|^{2} \\
& \quad \leq \lambda\left\|T_{a b} x-P y\right\|^{2}+(1-\lambda)\|P x-P y\|^{2}-\lambda(1-\lambda) \inf _{t \in G}\left\|T_{t} x-P x\right\|^{2} \\
& \quad=\left\|\lambda T_{a b} x+(1-\lambda) P x-P y\right\|^{2}+\lambda(1-\lambda)\left\|T_{a b} x-P x\right\|^{2}-\lambda(1-\lambda) \inf _{t \in G}\left\|T_{t} x-P x\right\|^{2} .
\end{aligned}
$$

Then it is easily seen that

$$
\begin{aligned}
& \inf _{s \in G} \sup _{t \in G}\left\|\lambda T_{t s} x+(1-\lambda) P x-P y\right\|^{2}-\lambda(1-\lambda) \inf _{b \in G a \in G}\left\|T_{a b} x-P x\right\|^{2} \\
& \quad \leq \sup _{b \in G} \inf _{a \in G}\left\|\lambda T_{a b} x+(1-\lambda) P x-P y\right\|^{2}-\lambda(1-\lambda) \inf _{t \in G}\left\|T_{t} x-P x\right\|^{2} .
\end{aligned}
$$

Since $P x \in L(\Im)$, we have

$$
\inf _{s \in G} \sup _{t \in G}\left\|\lambda T_{t s} x+(1-\lambda) P x-P y\right\|^{2} \leq \sup _{s \in G} \inf _{t \in G}\left\|\lambda T_{t s} x+(1-\lambda) P x-P y\right\|^{2} .
$$

Let

$$
h(\lambda)=\inf _{s \in G} \sup _{t \in G}\left\|\lambda T_{t s} x+(1-\lambda) P x-P y\right\|^{2} .
$$


56 Nonlinear ergodic theorems

Then for any $\varepsilon>0$, there exists $s_{2} \in G$ such that for all $t \in G$,

$$
\left\|\lambda T_{t s_{2}} x+(1-\lambda) P x-P y\right\|^{2} \leq h(\lambda)+\varepsilon
$$

and hence

$$
\left(\lambda T_{t s_{2}} x+(1-\lambda) P x-P y, P x-P y\right) \leq(h(\lambda)+\varepsilon)^{1 / 2}\|P x-P y\| \quad \forall t \in G .
$$

From $P x \in \overline{\operatorname{conv}}\left\{T_{t s_{2}} x: t \in G\right\}$, we have

$$
(\lambda P x+(1-\lambda) P x-P y, P x-P y) \leq(h(\lambda)+\varepsilon)^{1 / 2}\|P x-P y\| .
$$

Since $\varepsilon>0$ is arbitrary, this yields that

$$
\|P x-P y\|^{2} \leq h(\lambda) .
$$

That is,

$$
\|P x-P y\|^{2} \leq \inf _{s \in G} \sup _{t \in G}\left\|\lambda T_{t s} x+(1-\lambda) P x-P y\right\|^{2} .
$$

Now, one can choose an $s_{3} \in G$ such that $\left\|T_{t s_{3}} x-P x\right\| \leq M$ for all $t \in G$, where $M=1+\inf _{t \in G}\left\|T_{t} x-P x\right\|$. Then, we have

$$
\begin{aligned}
& \left\|\lambda T_{t s s_{3}} x+(1-\lambda) P x-P y\right\|^{2} \\
& \quad=\left\|\lambda\left(T_{t s s_{3}} x-P x\right)+(P x-P y)\right\|^{2} \\
& \quad=\lambda^{2}\left\|T_{t s s_{3}} x-P x\right\|^{2}+\|P x-P y\|^{2}+2 \lambda\left(T_{t s s_{3}} x-P x, P x-P y\right) \\
& \quad \leq M^{2} \lambda^{2}+\|P x-P y\|^{2}+2 \lambda\left(T_{t s s_{3}} x-P x, P x-P y\right) .
\end{aligned}
$$

It then follows from (3.6) and (3.12) that

$$
\begin{aligned}
2 \lambda \sup _{s \in G} \inf _{t \in G}\left(T_{t s} x-P x, P x-P y\right) \\
\quad \geq 2 \lambda \sup _{s \in G} \inf _{t \in G}\left(T_{t s s_{3}} x-P x, P x-P y\right) \\
\quad \geq \sup _{s \in G} \inf _{t \in G}\left\|\lambda T_{t s s_{3}} x+(1-\lambda) P x-P y\right\|^{2}-\|P x-P y\|^{2}-M^{2} \lambda^{2} \\
\quad=\sup _{s \in G} \inf _{t \in G}\left\|\lambda T_{t s} T_{s_{3}} x+(1-\lambda) P T_{s_{3}} x-P y\right\|^{2}-\|P x-P y\|^{2}-M^{2} \lambda^{2} \\
\geq\left\|P T_{s_{3}} x-P y\right\|^{2}-\|P x-P y\|^{2}-M^{2} \lambda^{2} \\
\quad=-M^{2} \lambda^{2} .
\end{aligned}
$$

Hence, we have

$$
\sup _{s \in G} \inf _{t \in G}\left(T_{t s} x-P x, P x-P y\right) \geq-\frac{1}{2} M^{2} \lambda .
$$

Letting $\lambda \rightarrow 0$, then we have

$$
\sup _{s \in G} \inf \left(T_{t s} x-P x, P x-P y\right) \geq 0 .
$$


Let $\varepsilon>0$, then there is $s_{4} \in G$ such that

$$
r\left(t s_{4}, x\right)<\varepsilon \quad \forall t \in G .
$$

For such an $s_{4} \in G$, from (3.16), we have

$$
\sup _{s \in G} \inf _{t \in G}\left(T_{t s} T_{s_{4}} x-P T_{s_{4}} x, P T_{s_{4}} x-P y\right) \geq 0
$$

and hence there is $s_{5} \in G$ such that

$$
\inf _{t \in G}\left(T_{t s_{5}} T_{s_{4}} x-P T_{s_{4}} x, P T_{s_{4}} x-P y\right)>-\varepsilon .
$$

Then, from $P T_{s_{4}} x=P x$, we have

$$
\inf _{t \in G}\left(T_{t s_{5} s_{4}} x-P x, P x-P y\right)>-\varepsilon .
$$

Similarly, from (3.16), we also have

$$
\sup _{s \in G} \inf \left(T_{t s} T_{s_{5} s_{4}} y-P T_{s_{5} s_{4}} y, P T_{s_{5} s_{4}} y-P x\right) \geq 0,
$$

and there exists $s_{6} \in G$ such that

$$
\inf _{t \in G}\left(T_{t s_{6} s_{5} s_{4}} y-P T_{s_{5} s_{4}} y, P T_{s_{5} s_{4}} y-P x\right) \geq-\varepsilon,
$$

that is,

$$
\inf _{t \in G}\left(P y-T_{t s_{6} s_{5} s_{4}} y, P x-P y\right) \geq-\varepsilon .
$$

On the other hand, from (3.20)

$$
\inf _{t \in G}\left(T_{t s_{6} s_{5} s_{4}} x-P x, P x-P y\right)>-\varepsilon .
$$

Combining (3.23) and (3.24), we have

$$
\begin{aligned}
-2 \varepsilon & <\left(T_{t s_{6} s_{5} s_{4}} x-T_{t s_{6} s_{5} s_{4}} y, P x-P y\right)-\|P x-P y\|^{2} \\
& \leq\left\|T_{t s_{6} s_{5} s_{4}} x-T_{t s_{6} s_{5} s_{4}} y\right\| \cdot\|P x-P y\|-\|P x-P y\|^{2} \\
& \leq\left(r\left(t s_{6} s_{5} s_{4}, x\right)+\|x-y\|\right) \cdot\|P x-P y\|-\|P x-P y\|^{2} \\
& \leq(\varepsilon+\|x-y\|) \cdot\|P x-P y\|-\|P x-P y\|^{2} .
\end{aligned}
$$

Since $\varepsilon>0$ is arbitrary, this implies $\|P x-P y\| \leq\|x-y\|$. The proof is completed.

Using Lemma 2.1, we have the following ergodic retraction theorem for asymptotically nonexpansive type semigroups.

TheOREM 3.2. Let $C$ be a nonempty subset of a real Hilbert space $H$ and let $\mathfrak{s}=$ $\left\{T_{t}: t \in G\right\}$ be a semitopological semigroup of asymptotically nonexpansive type mappings on $C$ such that $F(\Im) \neq \varnothing$. Then the following statements are equivalent:

(a) $\bigcap_{s \in G} \overline{\operatorname{conv}}\left\{T_{t s} x: t \in G\right\} \bigcap F(\Im) \neq \emptyset$ for each $x \in C$.

(b) There is a unique nonexpansive retraction $P$ of $C$ onto $F(\mathfrak{I})$ such that $P T_{t}=$ $T_{t} P=P$ for every $t \in G$ and $P x \in \overline{\operatorname{conv}}\left\{T_{t} x: t \in G\right\}$ for every $x \in C$. 
58 Nonlinear ergodic theorems

We denote by $B(G)$ the Banach space of all bounded real-valued functions on $G$ with supremum norm. Let $X$ be a subspace of $B(G)$ containing constants. Then, according to Mizoguchi-Takahashi [10], a real-valued function $\mu$ on $X$ is called a submean on $X$ if the following conditions are satisfied:

(1) $\mu(f+g) \leq \mu(f)+\mu(g)$ for every $f, g \in X$;

(2) $\mu(\alpha f)=\alpha \mu(f)$ for every $f \in X$ and $\alpha \geq 0$;

(3) for $f, g \in X, f \leq g$ implies $\mu(f) \leq \mu(g)$;

(4) $\mu(c)=c$ for every constant $c$.

The following corollaries are immediately deduced from Theorem 3.2.

Corollafry 3.3 (see [10]). Let $C$ be a closed convex subset of a Hilbert space $H$ and let $X$ be an $r_{s}$-invariant subspace of $B(G)$ containing constants which has a right invariant submean. Let $\mathfrak{s}=\left\{T_{t}: t \in G\right\}$ be a Lipschitzian semigroup on $C$ with $\inf _{s} \sup _{t} k_{t s}^{2} \leq 1$ and $F(\Im) \neq \emptyset$, where $k_{t}$ is the Lipschitzian constants. If for each $x, y \in C$, the function $f$ on $G$ defined by

$$
f(t)=\left\|T_{t} x-y\right\|^{2} \quad \forall t \in G
$$

and the function $g$ on $G$ defined by

$$
g(t)=k_{t}^{2} \quad \forall t \in G
$$

belong to $X$, then the following statements are equivalent:

(a) $\bigcap_{s \in G} \overline{\operatorname{conv}}\left\{T_{t s} x: t \in G\right\} \bigcap F(\Im) \neq \emptyset$ for each $x \in C$.

(b) There is a nonexpansive retraction $P$ of $C$ onto $F(\Im)$ such that $P T_{t}=T_{t} P=P$ for every $t \in G$ and $P x \in \overline{\operatorname{conv}}\left\{T_{t} x: t \in G\right\}$ for every $x \in C$.

COROLlafry 3.4 (see [7]). Let $C$ be a nonempty closed convex subset of a Hilbert space $H$ and let $\mathfrak{s}=\left\{T_{t}: t \in G\right\}$ be a continuous representation of a semitopological semigroup as nonexpansive mappings from $C$ into itself. If for each $x \in C$, the set $\bigcap_{s \in G} \overline{\operatorname{conv}}\left\{T_{t s} x: t \in G\right\} \bigcap F(\Im) \neq \emptyset$, then there exists a nonexpansive retraction $P$ of $C$ onto $F(\Im)$ such that $P T_{t}=T_{t} P=P$ for every $t \in G$ and $P x \in \overline{\operatorname{conv}}\left\{T_{t} x: t \in G\right\}$ for every $x \in C$.

Remark 3.5. By Theorem 3.2, many key conditions, in Corollaries 3.3 and 3.4, such as $C$ is convex closed subset and $\mathfrak{s}$ is continuous Lipschitzian semigroup, are not necessary.

\section{Acknowledgement}

The authors wish to acknowledge the financial support of the Korea Research Foundation made in the program year of 1998. 


\section{References}

[1] J.-B. Baillon, Un théorème de type ergodique pour les contractions non linéaires dans un espace de Hilbert, C. R. Acad. Sci. Paris Sér. A-B 280 (1975), no. 22, 1511-1514 (French). MR 51\#11205. Zbl 307.47006.

[2] , Quelques propriétés de convergence asymptotique pour les semi-groupes de contractions impaires, C. R. Acad. Sci. Paris Sér. A-B 283 (1976), no. 3, 75-78 (French). MR 54\#13655. Zbl 339.47028.

[3] H. Brézis and F. E. Browder, Remarks on nonlinear ergodic theory, Advances in Math. 25 (1977), no. 2, 165-177. MR 57\#1218. Zbl 399.47058.

[4] N. Hirano and W. Takahashi, Nonlinear ergodic theorems for nonexpansive mappings in Hilbert spaces, Kodai Math. J. 2 (1979), no. 1, 11-25 (English). MR 80j:47064. Zbl 404.47031.

[5] H. Ishihara and W. Takahashi, A nonlinear ergodic theorem for a reversible semigroup of Lipschitzian mappings in a Hilbert space, Proc. Amer. Math. Soc. 104 (1988), no. 2, 431-436 (English). MR 90g:47120. Zbl 692.47010.

[6] W. A. Kirk and R. Torrejón, Asymptotically nonexpansive semigroups in Banach spaces, Nonlinear Anal. 3 (1979), no. 1, 111-121 (English). MR 82a:47062. Zbl 411.47035.

[7] A. T. M. Lau, K. Nishiura, and W. Takahashi, Nonlinear ergodic theorems for semigroups of nonexpansive mappings and left ideals, Nonlinear Anal. 26 (1996), no. 8, 1411-1427 (English). MR 97b:47074. Zbl 880.47048.

[8] G. Li, Weak convergence and non-linear ergodic theorems for reversible semigroups of non-Lipschitzian mappings, J. Math. Anal. Appl. 206 (1997), no. 2, 451-464 (English). MR 98k:47139. Zbl 888.47046.

[9] G. Li and J. Ma, Nonlinear ergodic theorem for semitopological semigroups of nonLipschitzian mappings in Banach spaces, Chinese Sci. Bull. 42 (1997), no. 1, 8-11 (English). MR 98e:47110. Zbl 904.47063.

[10] N. Mizoguchi and W. Takahashi, On the existence of fixed points and ergodic retractions for Lipschitzian semigroups in Hilbert spaces, Nonlinear Anal. 14 (1990), no. 1, 69-80 (English). MR 91h:47071. Zbl 695.47063.

[11] W. Takahashi, A nonlinear ergodic theorem for an amenable semigroup of nonexpansive mappings in a Hilbert space, Proc. Amer. Math. Soc. 81 (1981), no. 2, 253-256 (English). MR 82f:47079. Zbl 456.47054.

[12] _ A nonlinear ergodic theorem for a reversible semigroup of nonexpansive mappings in a Hilbert space, Proc. Amer. Math. Soc. 97 (1986), no. 1, 55-58. MR 88f:47051.

[13] _ Fixed point theorem and nonlinear ergodic theorem for nonexpansive semigroups without convexity, Canad. J. Math. 44 (1992), no. 4, 880-887 (English). MR 93j:47091. Zbl 786.47047.

[14] W. Takahashi and P.-J. Zhang, Asymptotic behavior of almost-orbits of reversible semigroups of Lipschitzian mappings, J. Math. Anal. Appl. 142 (1989), no. 1, 242-249 (English). MR 90g:47121. Zbl 695.47062.

G. Li: Department of Mathematics, Yangzhou University, Yangzhou 225002, China

E-mail address: ligang@cims1.yzu.edu.cn

J. K. Kim: Department of Mathematics, Kyungnam University, Masan, Kyungnam 631701, KoREA

E-mail address: jongkyuk@kyungnam.ac.kr 


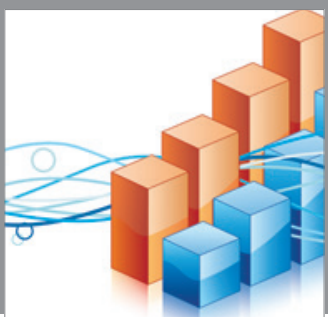

Advances in

Operations Research

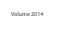

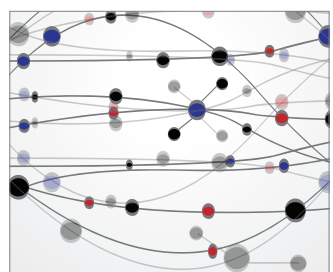

\section{The Scientific} World Journal
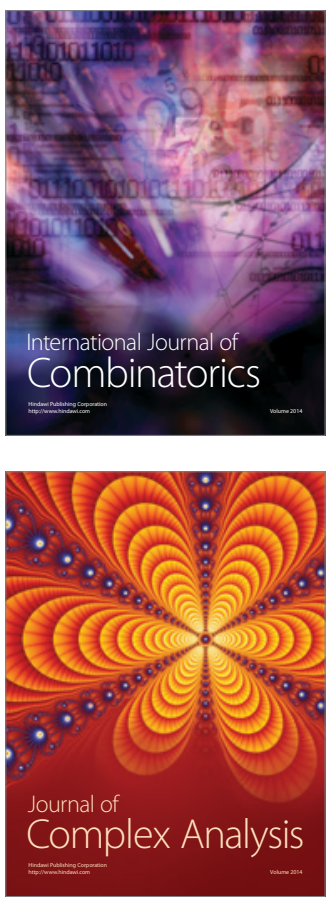

International Journal of

Mathematics and

Mathematical

Sciences
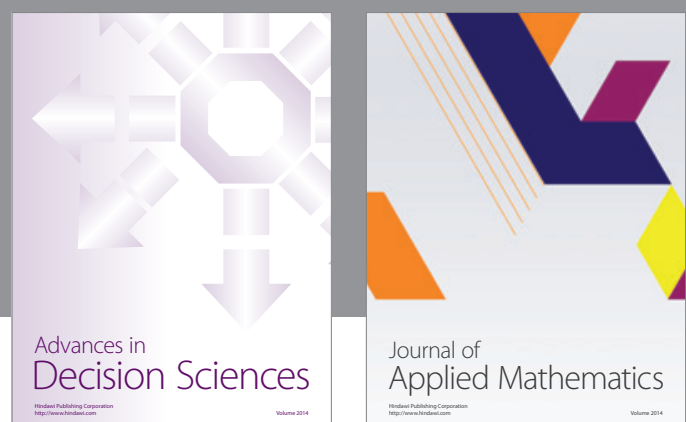

Journal of

Applied Mathematics
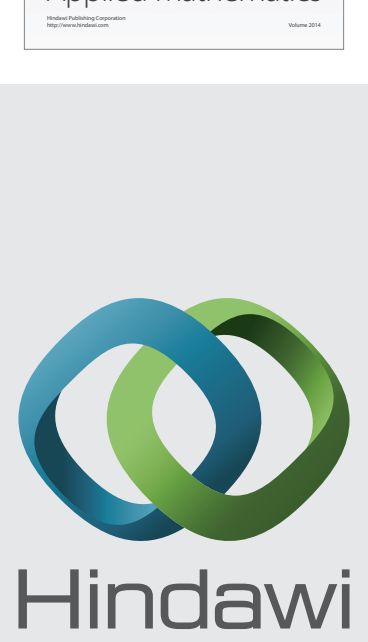

Submit your manuscripts at http://www.hindawi.com
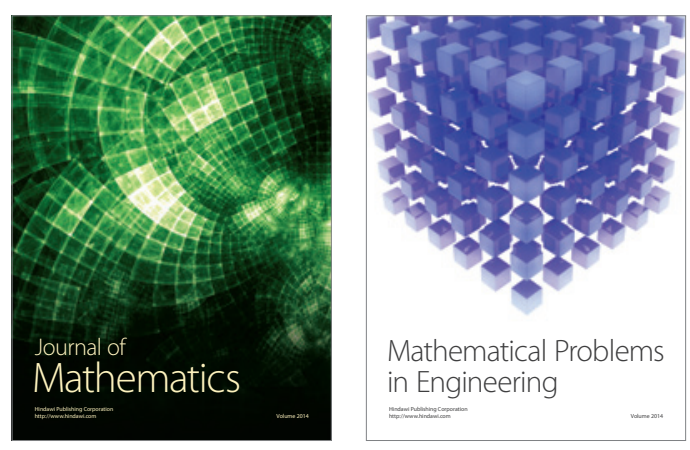

Mathematical Problems in Engineering
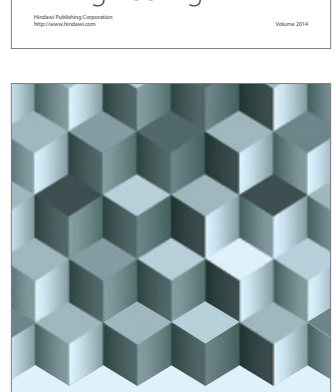

Journal of

Function Spaces
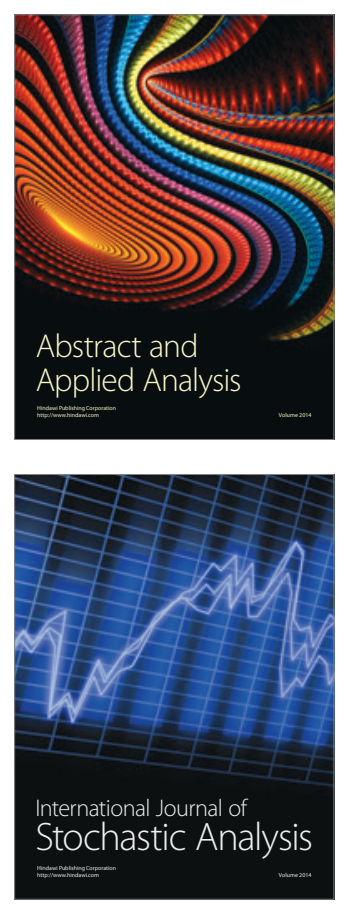

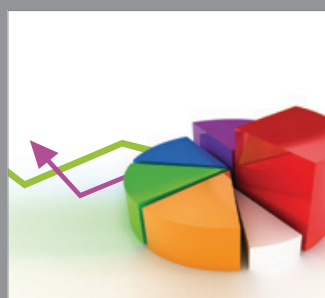

ournal of

Probability and Statistics

Promensencen
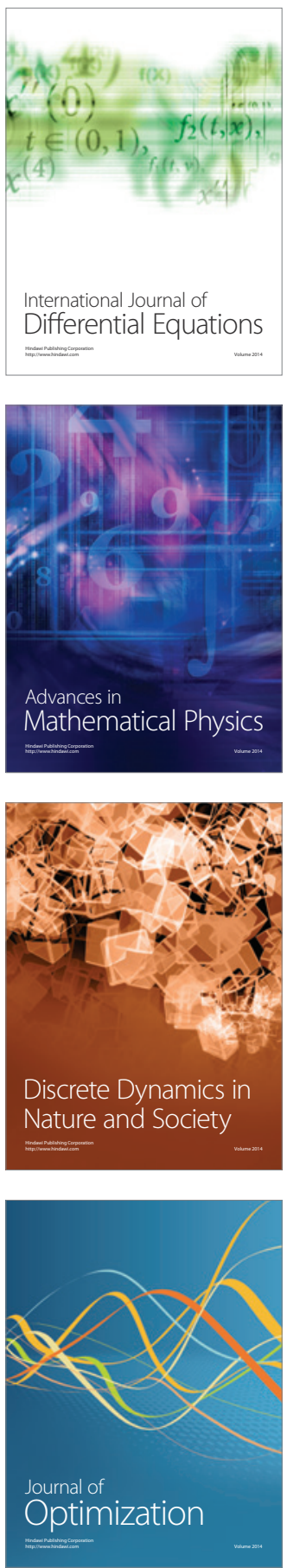